\title{
Trabalho interdisciplinar entre Universidade e Educação Básica para divulgação da Fisiologia: Relato de Experiência
} Interdisciplinary Work between University and Basic Education for the Publicising of Physiology: an Experience Report

Érica Maria Granjeiro ${ }^{1}$

1 Professora Adjuta. Universidade Estadual de Feira de Santana (UEFS), Brasil. E-mail:ericag@uefs.br

Recebido em: 31/03/2015 | Aprovado em: 04/02/2016

DOI: 10.12957/interag.2016.15871

\begin{abstract}
Resumo
Este artigo relata uma experiência de educação em saúde envolvendo o ensino de Fisiologia, conduzida por discentes de cursos na área da saúde da Universidade Estadual de Feira de Santana. A oficina direcionada para alunos da Educação Básica foi realizada na Universidade Estadual de Feira de Santana, Campus Feira de Santana/Bahia/Brasil, durante a Semana Nacional de Ciência e Tecnologia do ano de 2013. Várias atividades foram propostas direcionadas à prevenção das doenças cardiovasculares, principalmente, no que diz respeito à Hipertensão Arterial Sistêmica. A oficina por meio de um processo educativo participativo proporcionou ações de trabalho interdisciplinar, favorecendo as atividades de promoção de saúde e divulgação da Fisiologia tendo a escola como um espaço da atenção básica.
\end{abstract}

Palavras-chave: Ensino de fisiologia, Universidade, Escola.

Área temática: Metodologia e Experiência na Área da Educação, Promoção de Saúde.

Linha de extensão: Metodologia e Experiência na Área da Educação, Promoção de Saúde.

\begin{abstract}
This article reports a health educational experiment in Physiology lessons that was conducted by health science undergraduate students from Feira de Santana State University. The workshop was aimed at students in State Basic education schools and it took place at Feira de Santana State University, Feira de Santana Campus/Bahia/Brazil during the National Science and Technology Week in 2013. Several activities related to the prevention of cardiovascular diseases were proposed, mainly those related to hypertension. Through participatory educational process, the workshop provided interdisciplinary work, favoring the activities of health promotion and publicising of Physiology having the school as a space for Health Basic Attention.
\end{abstract}

Keywords: Physiology teaching, University, School.

\section{Introdução}

Estudo relata a importância de haver a real inserção da Fisiologia no contexto escolar, visto que isso ainda é pobremente trabalhado, especialmente na Educação Básica. ${ }^{1}$ Segundo Guyton \& $\mathrm{Hall}^{2}$, o objetivo da Fisiologia é explicar os fatores físicos e químicos que são responsáveis pela origem, desenvolvimento e progressão da vida. $\mathrm{O}$ conhecimento das causas dos fenômenos da vida no estado normal, por sua vez, nos ensinará a manter as condições normais da vida e a conservar a saúde. ${ }^{3}$ Entretanto, 
experiências mostram que transmitir informações a respeito do funcionamento do corpo humano, descrever as características das doenças, bem como elencar hábitos de higiene, não são suficientes para que os alunos desenvolvam atitudes de vida saudável. ${ }^{4}$ Dentro deste contexto, as oficinas podem ser ferramentas úteis, aumentando o interesse e instigando a busca pelo conhecimento.

Assim, no presente trabalho discentes e docentes de cursos de licenciatura e graduação da UEFS estiveram engajados na elaboração e implementação da I Oficina de Fisiologia Cardiovascular (OFC) da Universidade Estadual de Feira de Santana (UEFS) durante a Semana Nacional de Ciência e Tecnologia (SNCT) no ano de 2013. A SNCT, por sua vez, tem como finalidade principal mobilizar a população, em especial crianças e jovens, a respeito de temas e atividades de ciência e tecnologia $(\mathrm{C} \& \mathrm{~T}) \cdot{ }^{5} \mathrm{~A}$ coordenação nacional desta semana é de responsabilidade do Ministério da Ciência, Tecnologia e Inovação, por meio do Departamento de Popularização e Difusão de C\&T da Secretaria de C\&T para Inclusão Social. Esta campanha acontece anualmente, quando várias instituições de pesquisa, de ensino e municípios brasileiros realizam diversas ações em torno de uma temática de importância social. Especificamente no ano de 2013, a edição da SNCT abordou a temática "Ciência, Saúde e Esporte”. Assim, objetiva-se neste estudo, descrever a experiência em relação a esta oficina, envolvendo atividades educativas e serviços de saúde.

\section{Metodologia}

As atividades da I OFC-UEFS foram realizadas no dia 23 de outubro de 2013, durante a SNCT, na UEFS Campus Feira de Santana/Bahia/Brasil. Todas as ações propostas foram direcionadas a alunos da rede pública de Educação Básica, sendo utilizadas dinâmicas de caráter participativo. As ações foram coordenadas por docentes da Disciplina de Fisiologia Humana do Departamento de Ciências Biológicas (DCBIO) da UEFS e envolveram a participação voluntária de acadêmicos dos cursos de licenciatura em educação física e graduação em enfermagem, regularmente matriculados na disciplina de Fisiologia Humana (BIO407), bem como acadêmicos do curso de medicina da instituição. 
A metodologia utilizada para desenvolvimento das atividades consistiu em quatro etapas interligadas: Etapa 1: Aulas de Fisiologia Humana e rodas de leitura: Discentes dos cursos de educação física, enfermagem e medicina participaram de aulas teóricas e práticas de laboratório sobre Fisiologia Humana geral. Dentre os diversos temas abordados, destacamos as aulas de regulação a curto e a longo prazo da pressão arterial, contratilidade cardíaca, excitação rítmica do coração. A seguir, com o apoio e estímulo da Pró- Reitoria de Extensão da UEFS, foi feita a divulgação da SNCT junto aos diferentes cursos da instituição. Para tanto, foram realizadas aulas expositivas abordando a importância da SNCT para a comunidade em geral, levantando-se várias questões, incluindo: O que é SNCT? Qual a sua importância no contexto nacional e para a cidade de Feira de Santana? Quais temas estão relacionados à temática da SNCT do ano de 2013 "Ciência, Saúde e Esporte”? Além das aulas, foram realizadas rodas de leitura com a utilização de diversos artigos científicos relacionados a temas diversos de Fisiologia e Fisiopatologia, bem como problemas de Saúde Pública. Assim, foi proposta a realização da I OFC-UEFS elencando a Hipertensão Arterial Sistêmica (HAS) como foco para sua primeira edição. Etapa 2: Programação da Oficina: Uma vez selecionado o tema, iniciaram-se os planejamentos das atividades a serem realizadas durante a oficina. Com base na VI Diretrizes da Sociedade Brasileira de Hipertensão ${ }^{6}$, várias atividades foram propostas (Quadro 01). Esta etapa exigiu um trabalho intensivo de toda equipe proponente por meio de reuniões periódicas entre docentes e discentes da UEFS. Neste contexto, ressaltamos o fato de que o processo de seleção do tema e planejamento das atividades até a data de realização da oficina na UEFS demandou um período de seis meses (abril de 2013 a outubro de 2013). Etapa 3: Divulgação da Oficina: Em virtude de se tratar da primeira edição do evento na instituição, foi estabelecido diálogo com apenas uma escola pública com a finalidade de proporcionar uma atenção individualizada aos alunos. Para tanto, a equipe proponente realizou visita à Escola Municipal Chico Mendes da cidade de Feira de Santana, estado da Bahia. Assim, foi planejada a realização da oficina, durante a SNCT 2013, em turno oposto ao horário das aulas regulares na escola. Etapa 4: Implementação da oficina: Todas as atividades, as quais estão descritas no Quadro 01 e ilustradas na Figura 01, foram desenvolvidas pelos discentes sob orientação de um dos docentes. 
Título das atividades

1. Palestra para a comunidade

2. Mostra de Anatomia do Sistema Cardiovascular e Respiratório (SCR)

3. Medidas de Parâmetros Biológicos

4. Orientações sobre o Estilo Alimentar

5. Orientações sobre a prática de atividade física

\section{Detalhamento}

Nesta atividade foi discutida a problemática da HAS e sua relação com a obesidade e sedentarismo, em uma aula expositiva dialogada. Esta etapa teve como objetivo discutir com os participantes os diversos fatores envolvidos na gênese da HAS, tendo como foco a prevenção primária e a detecção precoce da doença.

Nesta atividade prática foram utilizadas peças anatômicas. Foram abordados aspectos específicos da anatomia do SCR, bem como práticas de ressuscitação cardíaca.

Nesta atividade foi realizada aferição da pressão arterial, frequência cardíaca, peso, altura, medida da circunferência abdominal, cálculo do índice de massa corporal dos participantes.

Nesta atividade foi abordado o padrão dietético DASH (Dietary Approaches to Stop Hypertension), rico em frutas, hortaliças, fibras, minerais e laticínios com baixos teores de gordura, o qual tem importante impacto na prevenção da hipertensão e/ou redução dos níveis pressóricos. ${ }^{7}$ Para despertar o interesse dos participantes, foram realizadas atividades práticas com demonstração de alimentos naturais e industrializados.

Por meio de pôsteres, cartazes e distribuição de panfletos, foi abordado o papel do exercício físico como uma das principais terapêuticas do paciente hipertenso, associado ao tratamento medicamentoso e às modificações de hábitos alimentares. ${ }^{8}$ Os alunos da educação básica também foram convidados a participar ativamente de dinâmicas de grupo com práticas de 
exercícios respiratórios, alongamentos e relaxamento.

Quadro 01: Descrição das atividades realizadas durante a I OFC-UEFS.
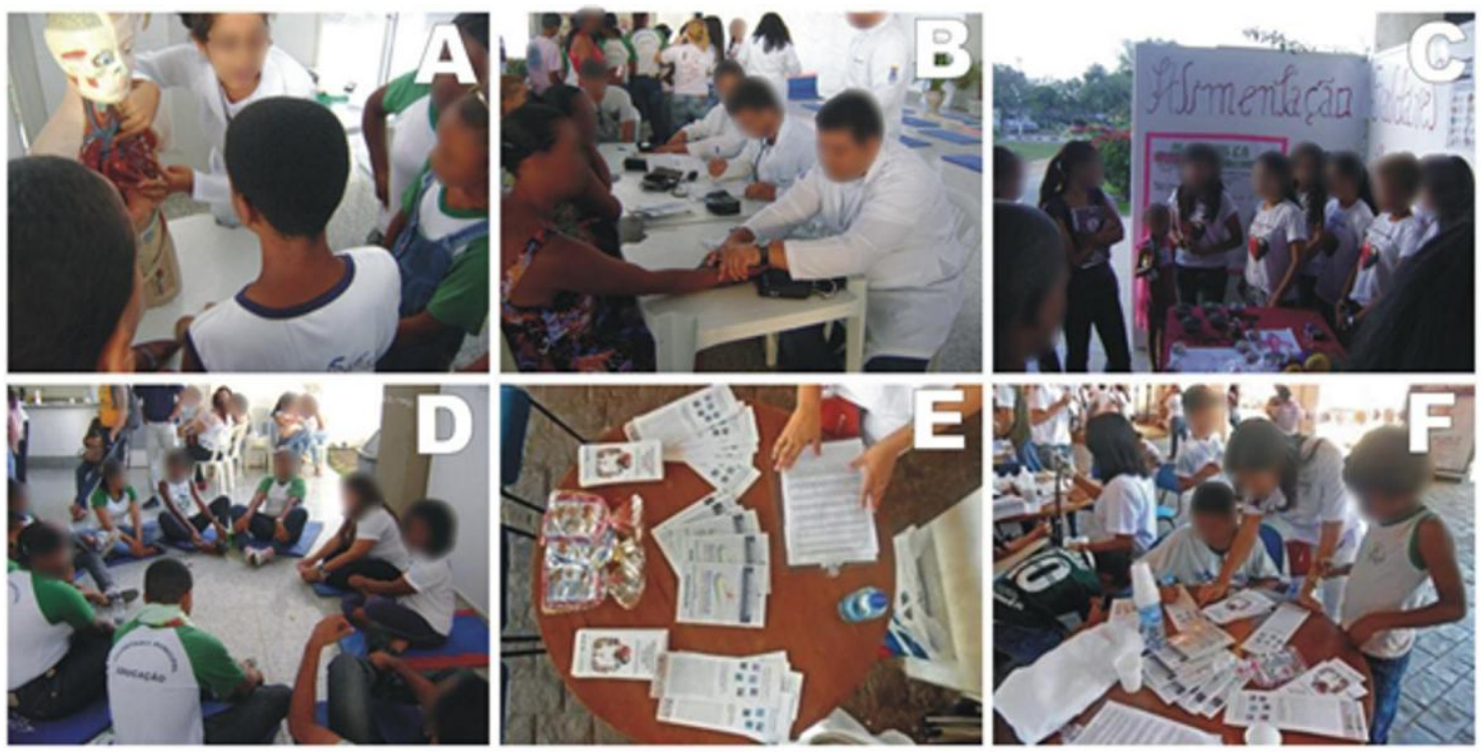

Figura 01. Imagens ilustrativas das atividades realizadas na IOFC -UEFS, SNCT 2013. 1A: Mostra de Anatomia; 1B: Aferição de Parâmetros Biológicos; 1C: Orientações sobre o Estilo Alimentar; 1D: Dinâmica de Grupo - Prática de Alongamento; 1E e 1F: Entrega de Panfletos e Folders.

\section{Resultados e Discussão}

Atualmente, a prática docente efetiva requer aprimoramento e atualização constante do professor que deve atuar como facilitador no processo de ensino-aprendizagem e não como transmissor de informações. ${ }^{9}$ Dentro desta perspectiva, no presente trabalho, discentes e docentes de cursos de licenciatura e graduação da UEFS estiveram engajados na elaboração e implementação da I OFC-UEFS, sendo estimulados a atuar como sujeitos reflexivos e ativos na vivência ensino-aprendizagem, e não como meros espectadores.

A I OFC-UEFS contou com a participação efetiva de 02 dois docentes, 45 discentes do curso de licenciatura em educação física, 45 discentes do curso de graduação em enfermagem e 6 discentes do curso de medicina da UEFS. Estiveram presentes 120 alunos da Escola Básica (primeiro e segundo ano do Ensino Médio). 
A inserção de estudantes da saúde incluindo médicos, enfermeiros, entre outros nas práticas educativas na promoção da saúde de escolares constitui-se uma ação pertinente e necessária. ${ }^{10}$ A partir do contato mais próximo com a realidade escolar, os discentes têm a oportunidade de desenvolver autonomia, senso crítico, reflexivo, criatividade e responsabilidade social, além da oportunidade de associar e até mesmo aplicar na prática vários conceitos adquiridos em sala de aula. Nesta oportunidade, chamamos a atenção para o papel das oficinas.

A I OFC-UEFS foi edificante, principalmente para os discentes, profissionais em formação, que dela participaram. As ações desenvolvidas forneceram possibilidades para os discentes, por meio de uma aprendizagem ativa, relacionar os conteúdos teóricos aprendidos em sala de aula com aqueles aplicados nas atividades práticas, tornando assim, o estudo significativo. Além disso, os mesmos tiveram a oportunidade de adquirir atitudes de liderança e trabalho em equipe, enquanto participantes e organizadores efetivos da oficina.

De fato, essa experiência despertou o interesse dos discentes na busca pelo conhecimento das causas dos fenômenos da vida no estado normal, quer dizer, a Fisiologia, além de colaborar para que os estudantes tornem-se melhores profissionais e promotores de saúde. A partir desta experiência, na busca de novos conhecimentos discentes têm buscado se inserir em programas institucionais que contemplam atividades educacionais inovadoras, incluindo atividades de pesquisa e extensão relacionadas à Fisiologia.

Com relação aos impactos das atividades sobre a Educação Básica, o trabalho realizado mostrou-se produtivo por diversos fatores. Primeiramente, pela receptividade da escola pública e abertura de um espaço para discussão de um tema que, muitas vezes, fica à margem do aprendizado escolar. Em segundo, pela aceitação da proposta pelos alunos da escola, os quais se mostraram extremamente interessados em discutir o tema. Em todas as atividades realizadas durante a oficina foi possível construir um espaço dialógico com os alunos, já que eles tiveram a oportunidade de manifestar suas opiniões. De fato, os alunos foram estimulados a atuar como sujeitos reflexivos e ativos na vivência ensino-aprendizagem realizada e não como meros espectadores. Neste contexto, a utilização de oficinas favorece um processo educativo participativo. ${ }^{11}$ 


\section{Considerações Finais}

A realização da I OFC-UEFS, durante a SNCT 2013, prevendo um diálogo entre universidade e escola, vem ao encontro dos anseios da Educação Básica, que busca parcerias com profissionais de saúde, objetivando o desenvolvimento de trabalhos que atendam à proposta dos temas transversais, aos projetos políticos de educação em saúde, e outras demandas que a escola recebe continuamente. Ademais, as atividades desenvolvidas no presente trabalho corroboram com as metas do Ministério da Educação (MEC) de incentivar práticas pedagógicas com abordagens interdisciplinares estruturadas pela relação entre teoria e prática. ${ }^{12}$

Considerado a importância da realização de atividades educativas e serviços de saúde direcionados a alunos da educação básica, esforços serão feitos para que novas edições sejam realizadas alcançando um maior número de escolas e alunos, estreitando, assim, o diálogo entre universidade e escola.

\section{Agradecimentos}

A Pró-Reitoria de Extensão da UEFS pelo apoio técnico. Aos docentes Profa. Ana Lúcia Nascimento (UEFS), Prof. Dr. Aristeu Silva Vieira (UEFS) e Profa Valéria Estânia Chaves (UFSJ) pela colaboração disponibilizada durante a realização da oficina e revisão final do texto, respectivamente. Aos acadêmicos envolvidos na execução das ações descritas neste artigo, bem como a direção e alunos da Escola Municipal Chico Mendes que participaram das ações.

\section{Referências}

1. DA ROSA, Liane Silveira; GONÇALVES, Rithiele; RODRIGUES, Taline; FAGUNDES, Flávia Manoela; TIEPPO, Kelly; ISOTON, Maicon; RIBEIRO, Patric da Silva; CARPES, Felipe Pivetta; MELLO, Elena Maria Billig; MELLO-CARPES, Pâmela Billig. Difusão da fisiologia através da capacitação de docentes da educação básica. Revista Ciência em Extensão, v.9, n.2, p.128-140, 2013.

2. GUYTON, Arthur C.; HALL, John E. Tratado de Fisiologia Médica.11.ed. Rio de Janeiro:Elsevier, 2006, $1115 \mathrm{p}$.

3. BERNARD, Claude. Introduction à l'étude de la medicine experimental. Paris: Flamarion, 1984. 
4. BRASIL. Parâmetros curriculares nacionais: terceiro e quarto ciclos: apresentação dos temas transversais. Secretaria de Educação Fundamental - Brasília: MEC/SEF, 1998.

5. Semana Nacional de Ciência e Tecnologia. Disponível em: http://semanact.mcti.gov.br/. Acesso em: 27 de novembro de 2015.

6. Sociedade Brasileira de Cardiologia / Sociedade Brasileira de Hipertensão /Sociedade Brasileira de Nefrologia. VI Diretrizes Brasileiras de Hipertensão. Arquivos Brasileiros de Cardiologia, v 95, n.1, supl.1, p. 1-51, 2010.

7. SACKS, Frank M.; SVETKEY, Laura P.; VOLLMER, William M.; APPEL, Lawrence J.; BRAY, George A.; HARSHA, David.; OBARZANEK, Eva; CONLIN, Paul R.; MILLER, Edgar R.; SIMONS-MORTON, Denise G.; KARANJA, Njeri; LIN, Pao-Hwa; AICKIN, Mikel; MOST-WINDHAUSER, Marlene M; MOORE, Thomas J.; PROSCHAN, Michael A., CUTLER, Jeffrey A. Effects on blood pressure of reduced dietary sodium and the Dietary Approaches to Stop Hypertension (DASH) diet. DASH-Sodium Collaborative. Research Group. The New England Journal of Medicine, v.344, p.3-10, 2001.

8. FLETCHER, Gerald F.; BALADY, Gary J.; AMSTERDAM, Ezra A.; CHAITMAN, Bernard; ECKEL, Robert; FLEG, Jerome; FROELICHER, Victor F.; LEON, Arthur S.; PIÑA, Ileana L.; RODNEY, Roxanne; SIMONS-MORTON, Denise A.; WILliAMS, Mark A.; BAZZARRE, Terr. Exercise standards for testing and training: a statement for healthcare professionals from the American Heart Association. Circulation, v.104, n.14, p. 1694-1740, 2001.

9. BARBOSA, Eduardo Fernandes; MOURA, Dácio Guimarães. Metodologias ativas de aprendizagem na Educação Profissional e Tecnológica. B. Tec. Senac, Rio de Janeiro, v.39, n.2, p.48-67, 2013.

10. SISTON AN; VARGAS LA. El enfermero em La escuela: Práticas educativas em La promoción de la salud de los escolares. Revista eletrónica semestral de enfermeria, v.6, n.11, novembro, 2007. Disponível em http://revistas.um.es/eglobal/article/view/409/521. Acesso em: 27 de novembro de 2015 .

11. DA SILVA, Doane Martins; ALvES, Marta dos Reis; DE SOUZA, Tatiane Oliveira; DUARTE, Ana Cristina Santos. Sexualidade na adolescência: Relato de Experiência. Revista de Enfermagem UFPE online, v.7, n.1, p.820-823, março, 2013.

12. Brasil. Ministério da Educação / Secretaria de Articulação com os Sistemas de Ensino (MEC). Planejando a Próxima Década Conhecendo as 20 Metas do Plano Nacional de Educação, 2014. 
